\title{
Associations Between Physical Fitness and Brain Structure in Young Adulthood
}

\author{
John R. Best ${ }^{1,2,3 *}$, Elizabeth Dao ${ }^{4}$, Ryan Churchill2 and Theodore D. Cosco ${ }^{1,2}$ \\ ${ }^{1}$ Gerontology Research Centre, Simon Fraser University, Vancouver, BC, Canada, ${ }^{2}$ Department of Gerontology, Simon \\ Fraser University, Vancouver, BC, Canada, ${ }^{3}$ Department of Psychiatry, University of British Columbia, Vancouver, BC, \\ Canada, ${ }^{4}$ Department of Radiology, University of British Columbia, Vancouver, BC, Canada
}

\section{OPEN ACCESS}

Edited by:

Maria António Castro,

College of Health Technology of Coimbra, Portugal

Reviewed by:

Carlos Cristi-Montero,

Pontificia Universidad Católica de Valparaíso, Chile

Snezana Smederevac,

University of Novi Sad, Serbia

*Correspondence: John R. Best john_best@sfu.ca; john.best@ubc.ca

Specialty section:

This article was submitted to Movement Science and Sport Psychology, a section of the journal Frontiers in Psychology

Received: 18 September 2020 Accepted: 30 October 2020 Published: 17 November 2020

Citation:

Best JR, Dao E, Churchill $R$ and Cosco TD (2020) Associations Between Physical Fitness and Brain

Structure in Young Adulthood.

Front. Psychol. 11:608049. doi: 10.3389/fpsyg.2020.608049
A comprehensive analysis of associations between physical fitness and brain structure in young adulthood is lacking, and further, it is unclear the degree to which associations between physical fitness and brain health can be attributed to a common genetic pathway or to environmental factors that jointly influences physical fitness and brain health. This study examined genotype-confirmed monozygotic and dizygotic twins, along with non-twin full-siblings to estimate the contribution of genetic and environmental factors to variation within, and covariation between, physical fitness and brain structure. Participants were 1,065 young adults between the ages of 22 and 36 from open-access Young Adult Human Connectome Project (YA-HCP). Physical fitness was assessed by submaximal endurance (2-min walk test), grip strength, and body mass index. Brain structure was assessed using magnetic resonance imaging on a Siemens 3T customized 'Connectome Skyra' at Washington University in St. Louis, using a 32-channel Siemens head coil. Acquired T1-weighted images provided measures of cortical surface area and thickness, and subcortical volume following processing by the YA-HCP structural FreeSurfer pipeline. Diffusion weighted imaging was acquired to assess white matter tract integrity, as measured by fractional anisotropy, following processing by the YA-HCP diffusion pipeline and tensor fit. Following correction for multiple testing, body mass index was negatively associated with fractional anisotropy in various white matter regions of interest (all $|z|$ statistics $>3.9$ ) and positively associated with cortical thickness within the right superior parietal lobe ( $z$ statistic $=4.6$ ). Performance-based measures of fitness (i.e., endurance and grip strength) were not associated with any structural neuroimaging markers. Behavioral genetic analysis suggested that heritability of white matter integrity varied by region, but consistently explained $>50 \%$ of the phenotypic variation. Heritability of right superior parietal thickness was large ( $75 \%$ variation). Heritability of body mass index was also fairly large ( $60 \%$ variation). Generally, $\frac{1}{2}$ to $\frac{2}{3}$ of the correlation between brain structure and body mass index could be attributed to heritability effects. Overall, this study suggests that greater body mass index is associated with lower white matter integrity, which may be due to common genetic effects that impact body composition and white matter integrity.

Keywords: heritability, environment, gray matter structure, white matter integrity, physical fitness, body composition 


\section{INTRODUCTION}

Associations between performance-based physical fitness and neuroimaging markers of brain health have been studied fairly extensively in the context of aging, and to a somewhat lesser extent in the context of childhood development. Previous studies have shown that physical fitness-most commonly cardiorespiratory fitness-positively correlates with gray matter (GM) thickness or volume in various regions of the cortex in children and older adults (Gordon et al., 2008; ChaddockHeyman et al., 2015; Wood et al., 2016; Williams et al., 2017; Esteban-Cornejo et al., 2019; Cadenas-Sanchez et al., 2020) and with hippocampal volume in older adults (Erickson et al., 2009; Cole et al., 2020). Other research has shown associations between physical fitness-again typically cardiorespiratory fitness-and higher diffusion-based white matter (WM) integrity (Marks et al., 2011; Tseng et al., 2013; Oberlin et al., 2015; Ding et al., 2018) or reduced lesion volume in older adults (Sexton et al., 2016). In children, one study found a weak association between upper-body strength and WM integrity in the frontal lobe (Rodriguez-Ayllon et al., 2020).

Relatedly, other research has looked at associations between body composition - most commonly body mass index (BMI) and neuroimaging markers of brain health in youth and older adults. Higher BMI has been associated with smaller GM volume in various cortical and subcortical regions in adolescence (Kennedy et al., 2016) and late adulthood (Ho et al., 2011), with reduced WM volume in adolescence (Kennedy et al., 2016), and with lower cerebral WM integrity in older adults (Marks et al., 2011). Still other studies have examined large age spans ranging from very early adulthood into older age. These studies, too, have found that BMI negatively correlated with WM integrity, especially in midbrain and brain stem tracts (Verstynen et al., 2012) and subregions of the corpus callosum (Stanek et al., 2011). Altogether, these findings have been used to argue that improving physical fitness and body composition might improve brain health, and in turn, promote cognitive performance during development (Chaddock-Heyman et al., 2015) and reduce cognitive impairment during aging (Erickson et al., 2014).

Analysis of links between physical fitness and body composition with neuroimaging markers of brain health specifically in early adulthood has been somewhat overlooked, despite acknowledgment that a lifespan perspective on brain health is warranted, in which risk factors for deterioration in brain health are potentially present across the lifespan (Moffitt et al., 2016; Williamson et al., 2018; Tucker-Drob, 2019). Evidence for such associations could provide the grounds for future research that explores whether intervening in young adulthood to promote fitness and body composition might alter long-term trajectories of brain health into older age.

The Young Adult Human Connectome Project (YA-HCP) is a large neuroimaging study of approximately 1200 women and men aged 22 to 36 (Van Essen et al., 2013), which offers an excellent data source for exploring links between physical fitness and body composition with various neuroimaging markers of brain health. Beyond collecting high-quality multi-model neuroimaging data, the YA-HCP collects behavioral data in a variety of domains, including physical fitness and body composition (Barch et al., 2013). Previous studies using the YAHCP have observed that higher BMI and lower submaximal cardiovascular endurance are associated with lower integrity within several WM tracts (Repple et al., 2018; Opel et al., 2019). Other YA-HCP analyses have shown that BMI correlates with cortical thickness, with the direction (negative versus positive) depending on the region and hemisphere (Vainik et al., 2018). In a recent study using the YA-HCP, we failed to observe an association between cortical thickness and either submaximal cardiovascular endurance or grip strength (Best, 2020). Like other studies in the field, our previous study was limited by focusing on only one type of brain structure (specifically, cortical thickness), despite knowledge that GM structure is distinct from WM structure, and even that cortical thickness is genetically and phenotypically distinct from cortical area (Winkler et al., 2010). As such, it is plausible that fitness and body composition might have different associations with different structural features of the brain. Further, although our previous study adjusted for participant height and weight in the regression analyses, BMI was not considered as an important predictor in its own right, and it is possible for fitness and body composition to have unique associations with brain structure.

Thus, the current study is motivated in part by the need for a comprehensive analysis involving multiple aspects of physical fitness and body composition along with various structural measures of brain health within a single sample with sufficient sample size to appropriately correct for multiple testing while maintaining reasonable statistical power. Using data from the YA-HCP, the current study estimated associations between submaximal cardiovascular endurance, grip strength, and BMI with cortical GM thickness and area, with subcortical GM volume, and with WM fractional anisotropy (FA), a commonlyused measure of WM integrity based on the flow of water molecules along WM tracts (Pierpaoli et al., 1996). Importantly, the default assumption made in most previous investigations is that associations between fitness/body composition and brain structure is linear across the range of scores; however, this may not necessarily be the case. Erickson et al. (2010) observed that the strongest association between physical activity and GM volume in older adults was at the highest quartile of physical activity, suggesting that a relatively large volume of physical activity might be necessary for detection of effects on brain structure. Whether such non-linear associations are observed for physical fitness or body composition is unknown, but the relatively large sample and wide distribution of fitness and body composition in the YA-HCP offers an opportunity to explore this possibility.

Assuming that links between physical fitness and brain structure exist in young adulthood, an important next step is to better understand the degree to which covariation in physical fitness and brain structure might be attributed to genetic versus environmental variation. The YA-HCP is wellsuited to enhance such understanding because of its inclusion of genotype-confirmed monozygotic and dizygotic twin pairs, along with full-sibling non-twin pairs. Assuming that genetic overlap approximates $100 \%$ in monozygotic twins and $50 \%$ in dizygotic 
and non-twin pairs, behavioral genetic analyses can be conducted to estimate the heritability and environmental contributions to variation in fitness and brain structure, as well as the covariation between the two (Grasby et al., 2017). Previous research has found a moderate heritability contribution to the correlation between cardiovascular endurance and executive function (Best, 2020) and between BMI and executive function, cortical thickness and medial temporal lobe volume (Vainik et al., 2018). Research in other samples has shown a moderate heritability contribution to the BMI-executive function correlation in youth (Wood et al., 2019) and a more modest heritability contribution to the cardiovascular fitness-intelligence association among young men enlisted for military service (Aberg et al., 2009). Altogether, these studies suggest at least a modest genetic component to correlations between physical fitness and body composition, on the one hand, and neuro-cognition, on the other. To followup on our primary analyses, the current study conducted a set of behavioral genetic analyses among a subset of twin and full-sibling, non-twin pairs involved in the YA-HCP to estimate the degree to which genetics and the environment underpin associations identified in the larger sample.

\section{MATERIALS AND METHODS}

\section{Study Design and Participants}

Participant data were provided by the open-access YA-HCP 1200 Subjects Data Release (Van Essen et al., 2013). For our primary analyses with the entire sample, 1,113 participants had valid FreeSurfer-processed 3T structural MRI data and 1,065 participants had valid FSL-processed diffusion imaging of WM data. For behavioral genetic analyses, a subset of the entire sample was used, which entailed twin or full-sibling, non-twin pairs who participated in the study. Twin zygosity was verified by genotyping. Full-sibling, non-twin pairs were identified by having identical mother and father identification numbers, but not being twins. Full-siblings with an age discrepancy $\geq 5$ years were excluded from behavioral genetic analyses. ${ }^{1}$ For behavioral genetic analyses involving WM FA values, data from 134 monozygotic twin pairs, 72 dizygotic twin pairs, and 290 full-sibling, non-twin pairs were available. For behavioral genetic analyses involving GM, data from 138 monozygotic twin pairs, 78 dizygotic twin pairs, and 313 full-sibling, non-twin pairs were used.

Participants were healthy adults within the age range of 22-36, who were drawn primarily from families that included twins in Missouri, United States. Families were excluded where individuals within the family had severe neurodevelopmental disorders (e.g., autism), neuropsychiatric disorders (e.g., schizophrenia) or neurological disorders (e.g., Parkinson's disease). Individuals were also excluded if they had illnesses thought to impact neuroimaging data quality (e.g., high

\footnotetext{
${ }^{1}$ It was possible for a given individual to be included in multiple sibling pairs, as there were families with up to six full siblings. In such instances, values were averaged across all pairs of the same type (i.e., DZ, MZ, non-twin full-sibling types) in which the given individual was involved, so as to avoid double-counting individuals. The vast majority of these involved non-twin, full-sibling pairs.
}

blood pressure or diabetes). Additional details on the participants and study design can be found elsewhere (Van Essen et al., 2013). Extensive details on all the measures included in the YAHCP study can be found at: https://wiki.humanconnectome.org. The original study was approved by the Washington University Institutional Review Board. All participants provided written informed consent. Approval for this secondary analysis was provided by the Institutional Review Board at Simon Fraser University (Title: "Genetic Contributions to Cognition and Physical Functioning in Young Adults: Analysis of the Human Connectome Project Study"; Study no: 2019s0471).

\section{Measures}

\section{Demographic and Other Covariate Variables}

Age in years, gender, race/ethnicity, years of completed education, and annual total household income were selfreported. Gait speed (meters per second) was assessed over 4-meters, starting from rest, and was included to account for variance in the endurance measure due to differences in gait.

\section{Physical Fitness}

Two physical fitness measures from the NIH Toolbox for Assessment of Neurological and Behavioral Function ('NIH toolbox') were completed on the first of the 2-day assessment schedule. The NIH toolbox includes psychometrically-validated measures of cognition and behavior, suitable for use across the human lifespan (Gershon et al., 2013; Weintraub et al., 2013). Details on its implementation within the YA-HCP can be found elsewhere (Barch et al., 2013).

Sub-maximal cardiovascular endurance was assessed by having participants walk as far as possible over a 2-min period of time, back-and-forth over a $50-\mathrm{ft}$ course. The total distance is recorded. Two trials were completed, with the trial with the longer distance walked used as the outcome of interest. Full force grip strength was measured with both hands using a Jamar Plus Digital dynamometer with the elbow bent to 90 degrees and arm against the trunk. Practice was conducted for each hand. Pounds of force for the dominant hand was used as the outcome of interest. Both fitness measures have shown good test-retest reliability (Intraclass correlation coefficient $[$ ICC] $\geq 0.88$ ) and convergent validity $(r \geq 0.77)$ with 'gold standard' measures of endurance and grip strength across adulthood (Reuben et al., 2013). Further, although grip strength is a more direct measure of upper body strength, it also strongly correlates with knee extension performance ( $r \geq 0.77$ ), a measure of lower body strength, suggesting grip strength may serve as a proxy for body strength more generally (Bohannon et al., 2012). Height was reported in inches and weight was reported in pounds; these values were converted to BMI with the following formula: $B M I=$ $703 \times \frac{\text { weight }(l b s)}{\text { height }(\text { in })^{2}}$. Using the guidelines from the United States Centers for Disease Control and Prevention', a BMI below 18.5 indicates underweight, 18.5 to 24.9 indicates normal or health weight, 25 to 29.9 indicates overweight, and 30 and above indicates an obese weight status.

\footnotetext{
${ }^{2}$ www.cdc.gov
} 


\section{Structural MRI Data Acquisition and Processing}

Gray matter imaging acquisition and processing was conducted by the YA-HCP research team with no additional processing on the freely-available data. Extensive details on the imaging protocol are found elsewhere, including details of additional scanning paradigms not included in the current study (Van Essen et al., 2012, 2013). All participants were scanned on a Siemens 3T customized 'Connectome Skyra' at Washington University in St. Louis, using a 32-channel Siemens head coil. Two T1-weighted and two T2-weighted scans were acquired at a spatial resolution of $0.7 \mathrm{~mm}$ isotropic voxels over two sessions. Each scan was evaluated by a trained rater for overall quality and only good/excellent scans were submitted to the structural pipelines, which used FreeSurfer 5.1 software and custom steps that combine the T1- and T2-weighted images for more accurate delineation of white and pial surfaces (Van Essen et al., 2013). Initial registration to MNI152 space was completed by FSL's FLIRT tool, followed by non-linear FNIRT to align subcortical structures. Cortical surface alignment was achieved using a surface-based registration using FreeSurfer, separately for each hemisphere. Average cortical thickness and surface area was obtained in 68 regions of interest (ROIs) across the two hemispheres. Additionally, subcortical GM volume was obtained in 14 ROIs in the limbic system.

Diffusion images were acquired at a spatial resolution of $1.25 \mathrm{~mm}$ isotropic voxels during a session including six runs (each $9 \mathrm{~min} 50 \mathrm{~s}$ in duration). Initial pre-processing of diffusion data was conducted by the YA-HCP research team and included intensity normalization across runs, 'TOPUP' susceptibility-induced distortion correction, and 'EDDY' eddy current and motion correction (Glasser et al., 2013). Subsequent processing was conducted by the current research team using standard tract-based spatial statistics in FSL (Smith et al., 2006). Fractional anisotropy (FA) images were registered to the FMRIB58 FA template and averaged to create a mean FA image. This image was used to create a WM skeleton using an FA threshold of 0.2. The Johns Hopkins University ICBM-DTI-81 WM atlas (Mori et al., 2008; Oishi et al., 2008), which consists of 48 ROIs, was used to mask the WM skeleton and average FA values for every ROI was obtained per participant.

\section{Statistical Analyses}

Analyses were conducted using R version $4.0 .2^{3}$ using the Rstudio environment ${ }^{4}$ ). An a priori power calculation was not conducted given that this is a secondary analysis of an existing dataset. For each analysis the maximum sample size possible was used. The first step was to regress the cortical thickness and surface area values, subcortical volumes and WM FA values on a set of covariates (age, gender, ethnicity, income, education, gait speed, and intracranial volume) and the physical fitness measures (grip strength, submaximal endurance, and BMI). All fitness measures

${ }^{3}$ r-project.org

${ }^{4}$ Rstudio.com were included together in the same regression model so as to model their independent associations with the neuroimaging outcomes. Generalized linear models fit by restricted estimated maximum likelihood were used for these regressions. To allow for non-linearity in the association of age and physical fitness with the outcome of interest, restricted cubic splines were specified with knots located at the 10th, 50th, and 90th percentile values of the age and physical fitness variables (Harrell, 2019a). A compound symmetry covariance matrix accounted for the clustering of participants by maternal ID (i.e., twin and nontwin siblings are not independent participants). A separate model was constructed for each outcome. All predictors were retained in the models regardless of the statistical significance of their Wald test. These regression models were constructed using rms version 6.0.1 (Harrell, 2019b). Control of the type I error rate was achieved by first allocating an equal portion of an overall $\alpha=0.05$ to each of the four outcome domains (i.e., cortical thickness and area, subcortical volume, and WM FA) and then to each of the three key predictors (endurance, strength, and BMI). Next, because outcomes were anticipated to correlate with one another (i.e., thickness in one region correlates with thickness in another region), the effective number of independent tests within each outcome domain was calculated using an approach that accounts for the degree of covariation among outcomes within the same domain (Nyholt, 2004). Thus, the $\alpha$ for the test of a specific predictor and region within a domain was calculated as:

$$
\alpha_{\text {corrected }}=\frac{0.05}{4 \times 3 \times \text { tests }_{\text {independent }}},
$$

where the estimated number of independent tests was 40.6, 57.8, 52.4, and 10.3 for FA, cortical thickness, cortical area, and subcortical volume measures, respectively. These corrected two-sided $\alpha$ values translated into a $z$ statistic threshold of 3.88 for the FA outcomes, 3.97 for cortical thickness outcomes, 3.95 for cortical area outcomes, and of 3.54 for the subcortical volumes.

The second step was to estimate the proportion of phenotypic covariance between neuroimaging measures and physical fitness that can be attributed to heritability versus environmental sources. These analyses were limited to monozygotic twin, dizygotic twin, and full-sibling non-twin pairs with an age discrepancy of less than 5 years. Using the package OpenMx (version 2.17.4), a bivariate Cholesky decomposition twin model was constructed (Grasby et al., 2017). The Cholesky decomposition models specified that phenotypic variation in neuroimaging and physical fitness could be attributed to heritability variance (A), shared environmental variance (C), a twin-specific shared environment (T), and non-shared environmental variance (E) (Figure 1). These sources of variation are estimated by assuming that monozygotic twins share $100 \%$ of heritability effects and that dizygotic twins and full-sibling non-twins each share $50 \%$ of heritability effects. The shared environmental effects are equivalent for all three groups; additionally, monozygotic and dizygotic twins have another shared environmental effect that non-twins do not have. To estimate the heritability and environmental 


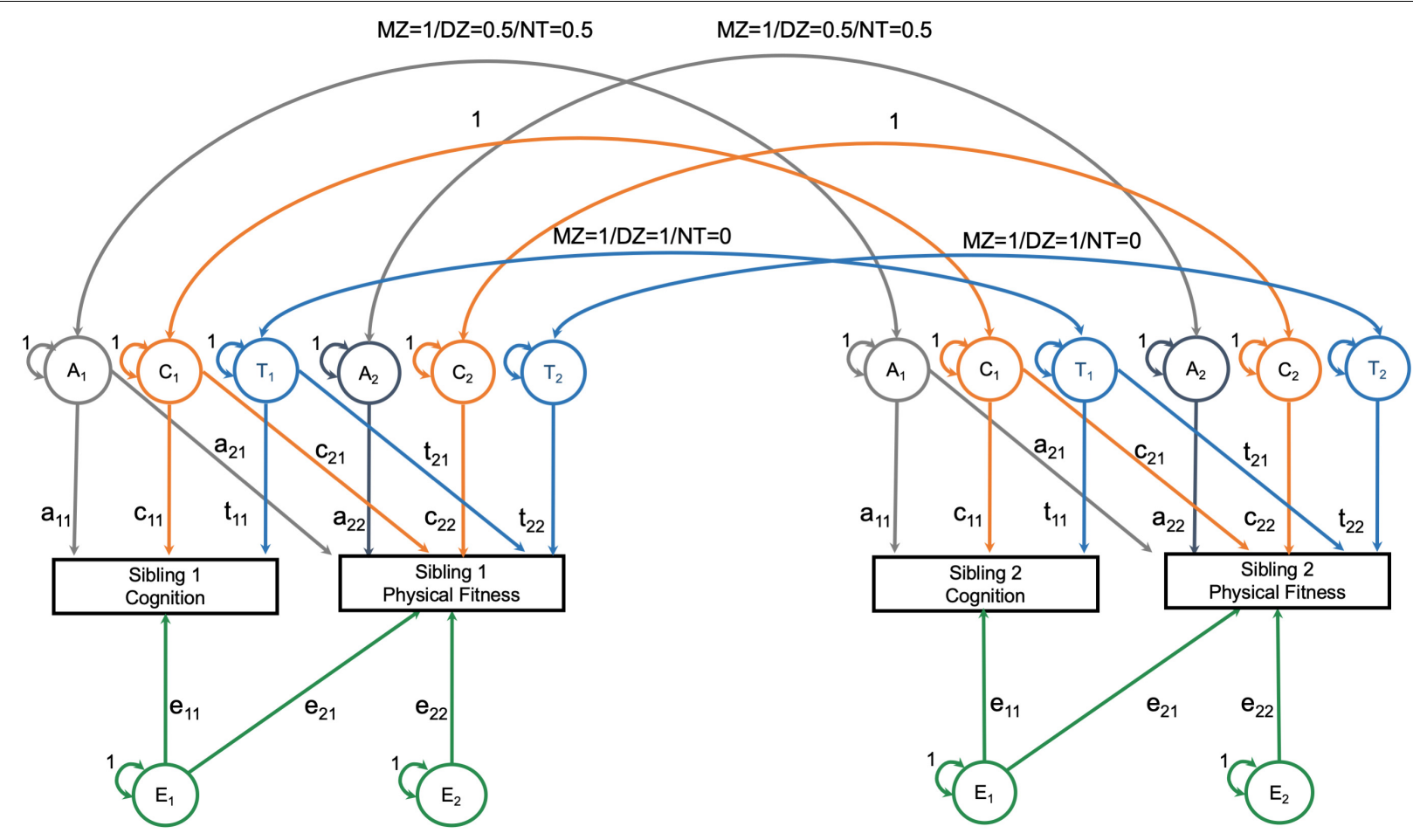

FIGURE 1 | Overview of bivariate Cholesky decomposition model.

contributions to the correlation between neuroimaging and physical fitness, the observed physical fitness variable is also allowed to load onto the latent heritability and environmental (shared, twin-specific, and non-shared) contributors to neuroimaging variation (Grasby et al., 2017). The result is the estimation of the variation in neuroimaging and physical fitness, as well as the covariation between neuroimaging and physical fitness. Prior to being entered into the Cholesky decomposition, neuroimaging and physical fitness variables were regressed on age (with non-linear restricted cubic spline effects), sex, race, education, annual income, and the interaction between age and sex in an ordinary least squares regression model (one model per outcome including all twins together).

The assumptions of equality of means and variances across twin order and zygosity were tested using the likelihood ratio test of nested models. A saturated model was fit first, in which all means and variances were allowed to vary freely across twins and zygosity. Next, means and variances were constrained to be equal across twin order, and then, across zygosity. Finally, the ACTwE model was estimated. To summarize the results of this model, standardize estimates and standard errors for each of the labeled paths in Figure 1 (e.g., "a11") are presented, as well as the proportion of variation of the cross-trait correlation that can be attributed to each of the components (i.e., A, C, Tw, or E). (Note. Total variance or covariance is the sum of the A, C, Tw, and $\mathrm{E}$ components. Covariance can then be converted into a correlation by dividing by the product of the standard deviation of the cognition measure and the standard deviation of the fitness measure).

All data used for the current study can be freely obtained by qualified registered researchers ${ }^{5}$ and the complete $\mathrm{R}$ analysis script can be found at https://osf.io/ykcm6/.

\section{RESULTS}

\section{Sample Characteristics and Initial Associations}

Demographic and physical fitness data on the full sample (i.e., all participants with GM and WM neuroimaging data) are summarized in Table 1 . Mean age was roughly 29 years and $46 \%$ were male. Nearly $70 \%$ of the sample was non-Hispanic white. Average BMI fell in the overweight range. Endurance, and to a lesser extent grip strength, were on average higher than the age-based population norms (mean $=100$ ) on which these measures were validated.

Simple bivariate correlations among age and physical fitness measures are shown in Table 2. Although gait speed was not a primary measure of interest, it is included as well. Age only weakly correlated with the other measures; endurance was modestly correlated with gait speed, grip strength and BMI $(|r| \sim 0.2-0.4)$.

\footnotetext{
${ }^{5}$ https://db.humanconnectome.org/
} 
TABLE 1 | Descriptive information on total sample.

\begin{tabular}{|c|c|c|c|}
\hline \multicolumn{4}{|l|}{ Variable } \\
\hline \multicolumn{2}{|l|}{ Sample size } & \multicolumn{2}{|c|}{1065} \\
\hline \multicolumn{2}{|l|}{ Age, years [mean $(S D)]$} & \multicolumn{2}{|c|}{$28.75(3.67)$} \\
\hline \multicolumn{2}{|l|}{ Sex, male (\%) } & \multicolumn{2}{|c|}{$490(46.0)$} \\
\hline \multicolumn{4}{|l|}{ Race/ethnicity (\%) } \\
\hline \multicolumn{2}{|l|}{ Asian or Pacific Islands } & \multicolumn{2}{|c|}{$64(6.0)$} \\
\hline \multicolumn{2}{|l|}{ Black/African American } & \multicolumn{2}{|c|}{$148(13.9)$} \\
\hline \multicolumn{2}{|l|}{ Hispanic/Latino } & \multicolumn{2}{|c|}{$94(8.8)$} \\
\hline \multicolumn{2}{|l|}{ Non-Hispanic, White } & \multicolumn{2}{|c|}{$734(68.9)$} \\
\hline \multicolumn{2}{|l|}{ Other } & \multicolumn{2}{|c|}{$25(2.3)$} \\
\hline \multicolumn{4}{|c|}{ Annual household income (\%) } \\
\hline \multicolumn{2}{|l|}{$<\$ 10 \mathrm{k}$} & \multicolumn{2}{|c|}{$72(6.8)$} \\
\hline \multicolumn{2}{|l|}{ 10-20k } & \multicolumn{2}{|c|}{$82(7.7)$} \\
\hline \multicolumn{2}{|l|}{ 20-30k } & \multicolumn{2}{|c|}{$136(12.8)$} \\
\hline \multicolumn{2}{|l|}{$30-40 k$} & \multicolumn{2}{|c|}{$126(11.9)$} \\
\hline \multicolumn{2}{|l|}{$40-50 k$} & \multicolumn{2}{|c|}{$106(10.0)$} \\
\hline $50-75 \mathrm{k}$ & & & (21.2) \\
\hline $75-100 k$ & & & $(13.8)$ \\
\hline$\geq 100 k$ & & & $(15.7)$ \\
\hline Years of education ( & & & \\
\hline 11 or less & & & $(3.4)$ \\
\hline 12 & & & $(13.7)$ \\
\hline 13 & & & $(6.1)$ \\
\hline 14 & & & (12.3) \\
\hline 15 & & & $(6.1)$ \\
\hline 16 & & & $(42.7)$ \\
\hline 17 or more & & & $(15.7)$ \\
\hline Gait speed, meters per & ond [mean $(S D)]$ & & $(0.20)$ \\
\hline Body mass index [mea & & & $(5.11)$ \\
\hline Endurance, age norme & ean $(S D)]$ & 10 & (13.94) \\
\hline Grip strength, age norr & [mean (SD)] & 10 & (20.12) \\
\hline Age & Endurance & BMI & Gait \\
\hline Age & -0.09 & 0.09 & 0.04 \\
\hline Endurance & 0.28 & -0.35 & 0.24 \\
\hline Strength & 1 & 0.15 & -0.02 \\
\hline BMl & & 1 & -0.09 \\
\hline Gait & & & 1 \\
\hline
\end{tabular}

\section{Physical Fitness and Structural Neuroimaging Markers White Matter Fractional Anisotropy}

Twelve of the possible $48 \mathrm{WM}$ ROIs were associated with BMI with $z$ test statistic that exceeded the multiplicity-corrected threshold. These ROIs are summarized in Table $\mathbf{3}$ and are arranged from largest to smallest $|z|$ statistic. Identified regions included midbrain tracts (bilateral cerebral peduncle and pontine crossing), bilateral fornix and hippocampal portion of the cingulum, and left sagittal stratum, among others. In all cases, greater BMI was associated with lower FA values.
TABLE 3 | Summary of effects of body mass index on FA values.

\begin{tabular}{llccc}
\hline $\begin{array}{l}\text { White matter region of } \\
\text { interest }\end{array}$ & Abbr. & Effect & S.E. & Z stat \\
\hline Cerebral peduncle left & & & & \\
Fornix cres left & CpL & -0.0081 & 0.0011 & -7.7 \\
Fornix cres right & FCL & -0.01 & 0.0016 & -6.5 \\
Cerebral peduncle right & FcR & -0.0092 & 0.0016 & -5.9 \\
$\begin{array}{l}\text { Pontine crossing tract } \\
\text { Sagittal stratum left }\end{array}$ & CpR & -0.0063 & 0.0011 & -5.8 \\
$\begin{array}{l}\text { Cingulum hippocampus right } \\
\text { Retrolenticular part internal }\end{array}$ & SsL & -0.0074 & 0.0015 & -5 \\
capsule right & RpicR & -0.0062 & 0.0014 & -4.4 \\
$\begin{array}{l}\text { Cingulum hippocampus left } \\
\text { Corticospinal tract left }\end{array}$ & ChL & -0.0082 & 0.0019 & -4.3 \\
$\begin{array}{l}\text { Superior fronto-occipital } \\
\text { fasciculus right }\end{array}$ & CtL & -0.0073 & 0.0018 & -4.1 \\
Inferior cerebellar peduncle & SffR & -0.0057 & 0.0015 & -3.9 \\
right & & & & -4.7 \\
\hline
\end{tabular}

Abbr., abbreviation. S.E., standard error. Z stat, Z statistic.

Marginal plots of the association between BMI and these WM ROIs are shown in Figure 2, which account for the covariates and other fitness measures. In some instances, the association between BMI and FA was fairly constant across the range of BMI values (e.g., left and right fornix), whereas in other instances, the association appeared to dissipate somewhat with more extreme BMI values (e.g., left and right cerebral peduncle).

Neither endurance nor grip strength had associations with the WM ROIs with $z$ statistics beyond the threshold. Comprehensive results for all WM ROIs can be found in Section "Comprehensive White Matter Fractional Anisotropy Results" for the Supplementary Material.

\section{Gray Matter Structure}

Body mass index was positively associated with right superior parietal thickness (see Table 4). As shown in the marginal plot in Figure 3 this association was strongest as BMI increased from the normal to the overweight range and then weakened somewhat with more extreme BMI values. No additional fitness associations with GM structure (whether thickness, area, or subcortical volume) had significant $z$ statistics. Comprehensive results for all GM ROIs can be found in Section "Comprehensive Gray Matter Results” for the Supplementary Material.

\section{Bivariate Behavioral Genetic Analysis of Fitness and Brain Structure}

Table 5 presents descriptive data for the demographic and physical fitness measures for each of the three sibling pair types, which shows similarities between participating siblings and among the three sibling pair types. Based on the results of the larger sample, bivariate genetic models were estimated between BMI and 12 WM ROIs and right superior parietal GM thickness. The full ACTwE model showed small estimates with large standard errors for the shared environmental and twin-specific environmental effects. As has been done in previous research (Vainik et al., 2018; Wood et al., 2019), 


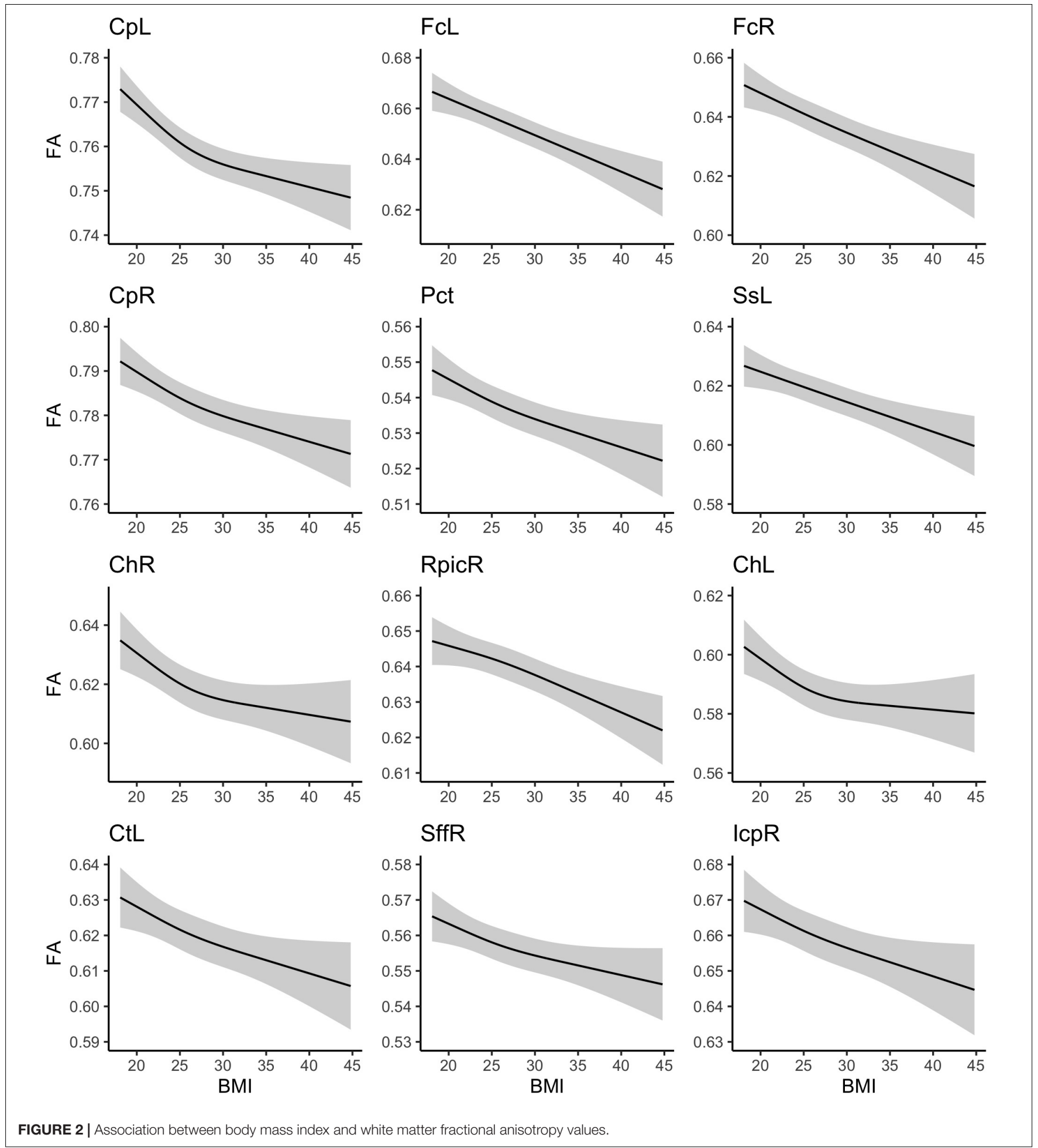

the loadings and cross-loadings for these two effect were fixed to zero, resulting in a simpler AE bivariate model. Across brain regions, this simpler AE model did not result in a worsening in fit according to likelihood ratio tests (smallest likelihood ratio test $p$-value was 0.14 across the 12 WM and 1 GM ROIs), and therefore, is described below.
Comparison between ACTwE and AE models and tests for the assumptions of equality of means and variances can be found in Section "Behavioral Genetic Model Comparisons" of the Supplementary Material, where we provide $-2 * \log$ likelihood values, degrees of freedom, and Akaike Information Criterion for each model. 
TABLE 4 | Summary of effects of body mass index on GM cortical thickness.

\begin{tabular}{llcc}
\hline Outcome & Effect & S.E. & $\boldsymbol{Z}$ stat \\
\hline R Superior parietal & 0.022 & 0.0047 & 4.6 \\
\hline
\end{tabular}

S.E., standard error. Z stat, Z statistic.

\section{White Matter Fractional Anisotropy}

Initial bivariate correlations across siblings, across traits, and across siblings and traits for the three sibling pair types are shown in Figure 4 for the 12 WM ROIs. These provide a helpful preview to the formal behavioral genetic analyses presented below. Body mass index was strongly correlated among MZ twins $(r \sim 0.61)$, and was weakly correlated among DZ twins and non-twin siblings $(r \sim 0.20-0.27)$. This suggests an important role of heritability in BMI variation. Similarly, WM FA was most strongly correlated within MZ twins for all 12 ROIs, also suggesting an important role of heritability for WM integrity. Whether DZ twins or non-twin siblings had the next strongest correlation varied by ROI, thereby suggesting inconsistency with regard to twin-specific versus a more general shared environmental effect. Cross-trait

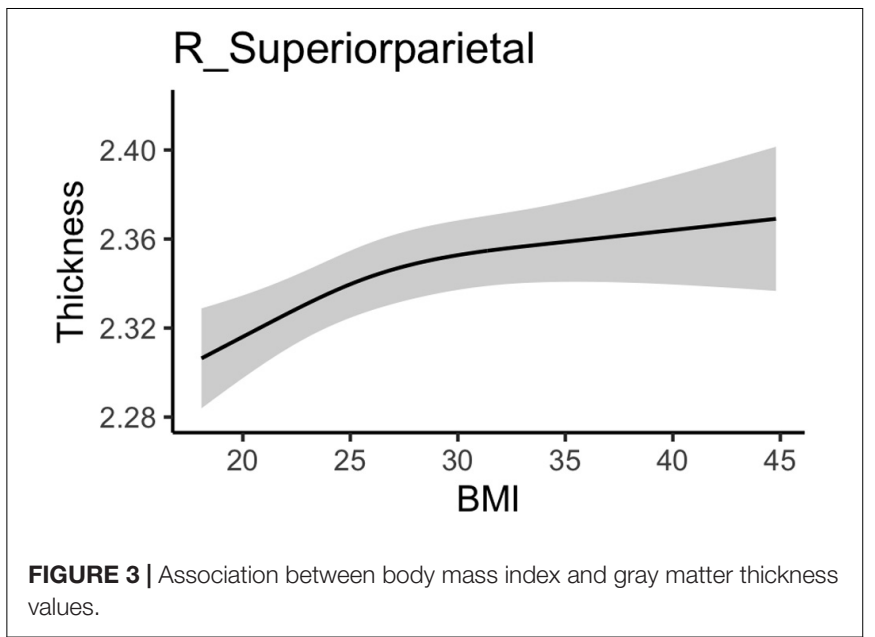

and cross-trait/cross-sib ('ct/cs' in Figure 4) correlations also varied somewhat depending on the WM ROI, though in general, the strongest negative correlation was among MZ twins and further, the cross-trait/cross-sibling correlation was

TABLE 5 | Descriptive information on each sibling pair type.

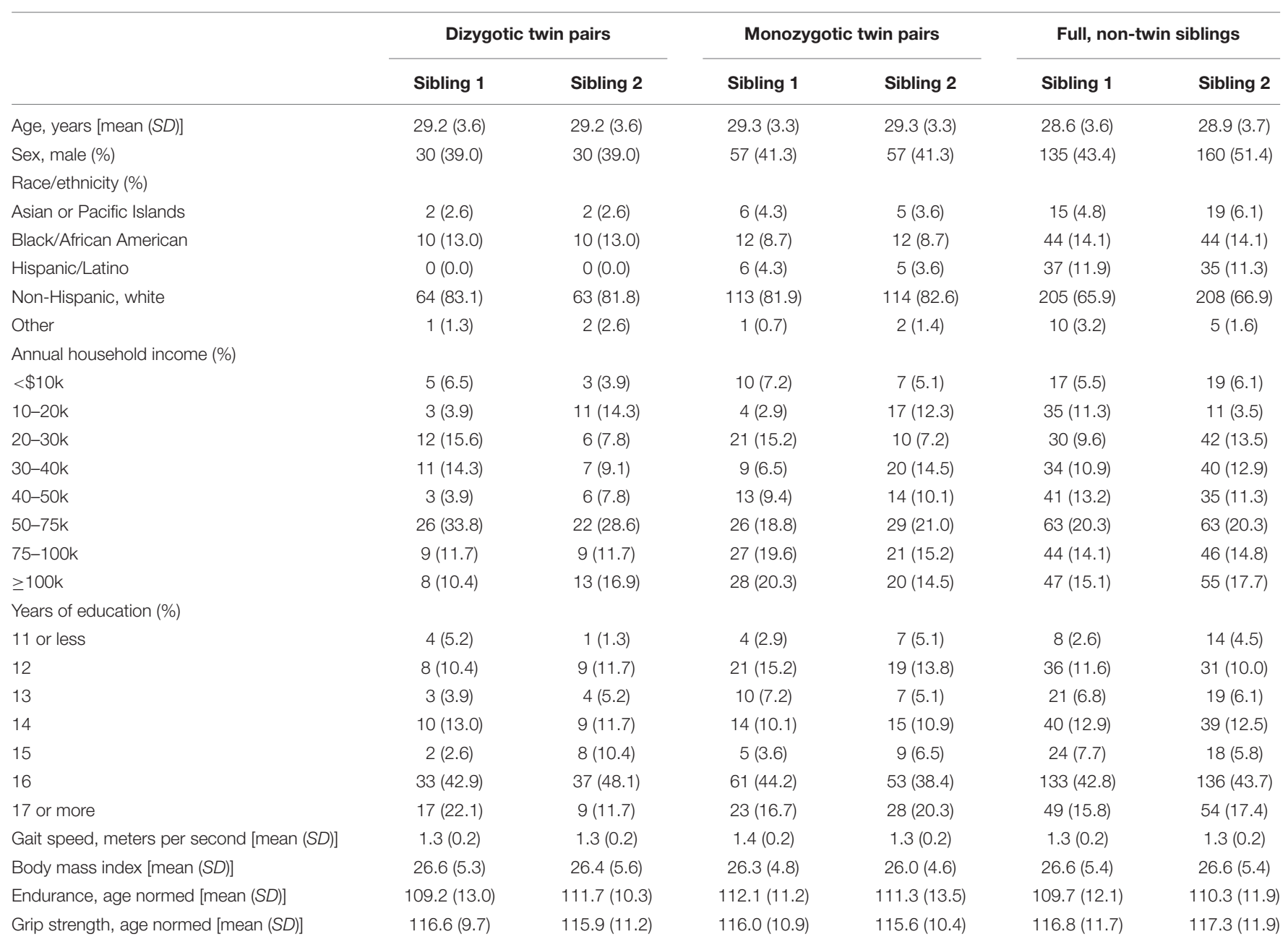




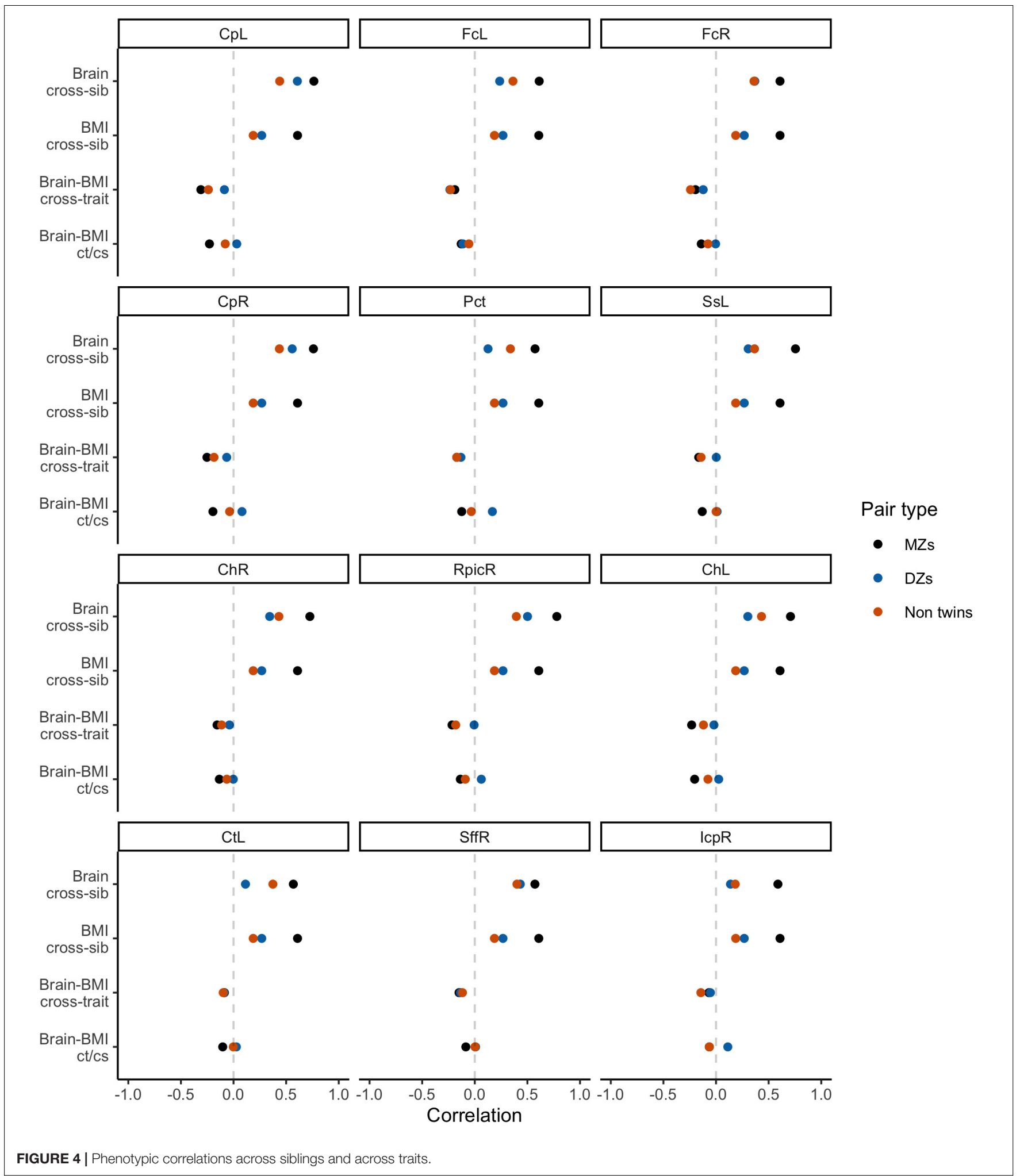

nearly as strong as the cross-trait correlation among MZ twins. This implies at least some role of heritability in the correlation between BMI and FA values; however, due to these correlations being generally quite modest (i.e., at most $r \sim-0.30)$, the absolute role of heritability is also anticipated to be modest.

Standardized estimates and 95\% confidence intervals for each of the paths in the bivariate Cholesky decomposition 
model are provided for each of the 12 WM ROIs in Figure 5. Following on the correlations described directly above, there were strong heritability loadings on WM FA variance (path a11) and BMI variance (a22). Similarly, there were strong, precisely estimated environmental effects for FA variance (e11) and BMI variance (e22). The point estimate for the genetic cross-loading (a21) was negative and modest $(\beta \sim-0.20)$; in some instances, the confidence interval for this estimate crossed

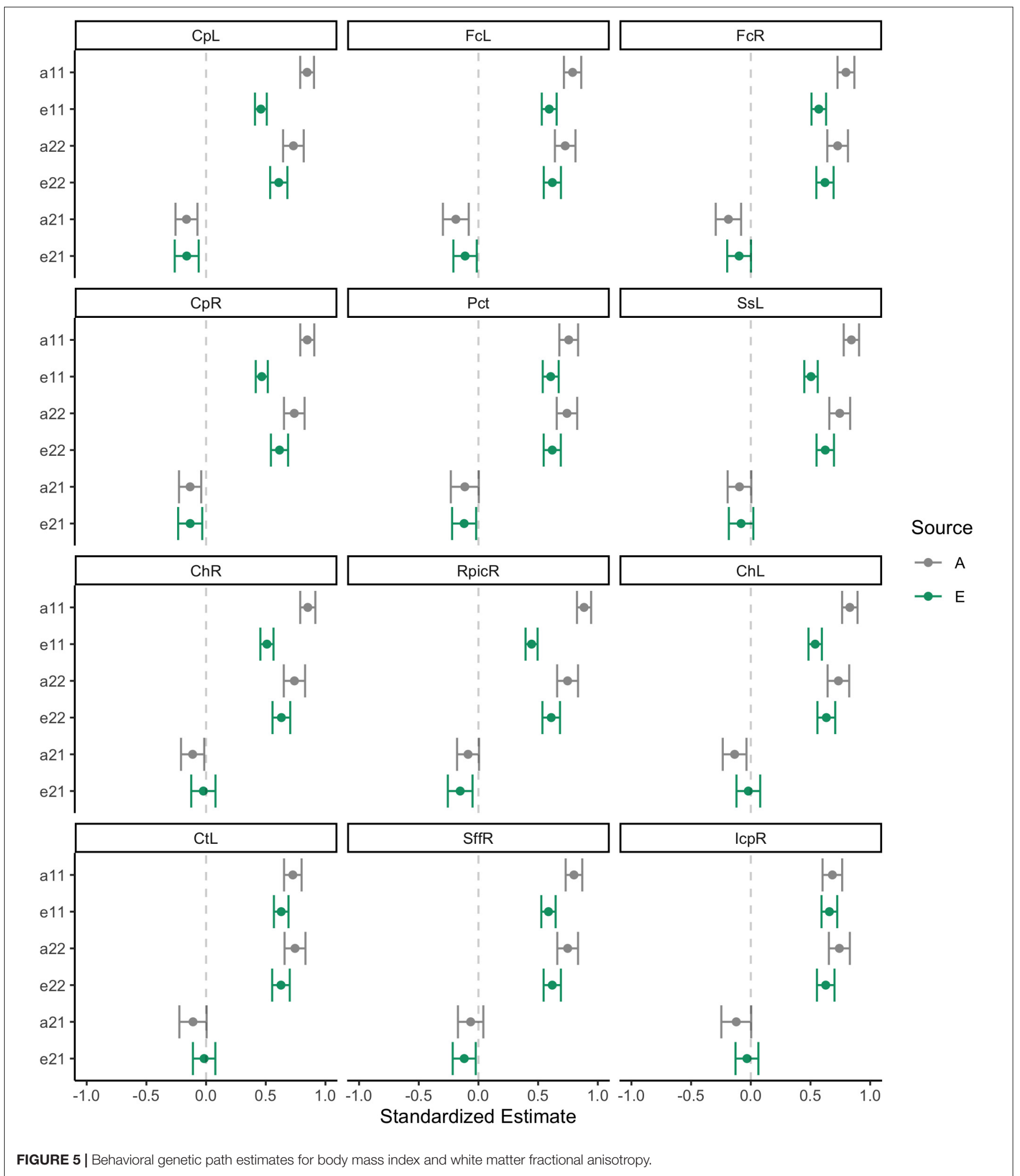


into positive numbers, suggesting some uncertainty about the nature of this effect. The point estimate for the environmental cross-loading (e21) was also quite small, generally smaller in magnitude than the genetic cross-loading. The confidence interval for this estimate was fairly narrow, but at times crossed into positive numbers.

These standardized estimates were then converted into variances and used to visualize how much of the variation in FA values and BMI could be attributed to each of the four sources. For example, in the AE model, WM FA variance is defined as below:

$$
\operatorname{Var}_{F A}=\operatorname{Var}_{F A_{A}}+\operatorname{Var}_{F A_{E}}
$$

For WM FA, each of the variance components is the square of the respective standardized loading. For example,

$$
\operatorname{Var}_{F A_{A}}=a_{11}^{2}
$$

For BMI, each of the variance components is the sum of the respective squared cross-loading and the squared endurancespecific standardized loading. For example,

$$
\operatorname{Var}_{B M I_{A}}=a_{21}^{2}+a_{22}^{2}
$$

The estimates from the AE model can also be used to partition the phenotypic correlation into A and E components. For example, the portion of the correlation attributed to heritability can be estimated by first estimating the covariance:

$$
\operatorname{Cov}_{A}=a_{11} \times a_{21}
$$

And then converting that value into a correlation:

$$
\operatorname{Cor}_{A}=\frac{\operatorname{Cov}_{A}}{\left(\operatorname{Var}_{F A_{A}}+\operatorname{Var}_{F A_{E}}\right)^{\frac{1}{2}} \times\left(\operatorname{Var}_{B M I_{A}}+\operatorname{Var}_{B M I_{E}}\right)^{\frac{1}{2}}}
$$

Proportions of the variances and of correlations are plotted in Figure 6. The decomposition of BMI variance is roughly identical across models (as expected) and shows that heritability contributed to roughly $50 \%$ of variance. Similarly, heritability contributed to between 50 and $78 \%$ variance in WM FA, depending on the ROI. Environmental factors, by design, explained the remainder of the variance. Covariances were converted to correlations and are also shown in Figure 6. As expected, the total phenotypic correlation was modestly negative, ranging between -0.20 and -0.25 . Generally, heritability accounted for $>\frac{1}{2}$ of this correlation. Section "White Matter Fractional Anisotropy" of the Supplementary Material shows the analysis of these 12 WM ROIs using the ACTwE model.

\section{Gray Matter Structure}

All of the above behavioral genetic analyses were repeated on the right superior parietal thickness, with the results summarized in Figure 7. As can be seen across the panels, there was a strong heritability effect for cortical thickness in addition to BMI (as described above). Analysis of the contributors to the correlation between BMI and cortical thickness in this region showed small positive effects for heritability (a21) and environment (e21) with confidence intervals that slightly cross into negative territory (see panel B). Heritability was estimated to contribute to $74 \%$ of variation of the variance in GM thickness and to slightly over $\frac{1}{2}$ of the small positive phenotypic correlation (panel C). Section "Gray Matter Structure" of the Supplementary Material shows the analysis of this GM ROI using the ACTwE model.

\section{DISCUSSION}

In this large study of young adults, we observed that BMI, but not performance-based measures of fitness, negatively correlated with WM integrity in various WM tracts. Except for a single positive association between BMI and cortical thickness in the right superior parietal lobe, we failed to observe associations between either physical fitness or BMI with cortical or subcortical GM structure. Along with a small set of previous studies (Aberg et al., 2009; Repple et al., 2018; Williamson et al., 2018; Opel et al., 2019; Repple et al., 2019), we bring attention to the fact that modifiable risk factors, such as BMI, correlate with brain health even in young adulthood. Our results support a lifespan perspective on neurocognitive aging, in which it may be fruitful to intervene many decades prior to the onset of cognitive impairment to effectively reduce negative impacts of cognitive aging (Moffitt et al., 2016; Tucker-Drob, 2019).

\section{Body Composition and Neurocognitive Health}

These results add to a growing literature associating high BMI and obesity with lower neurocognitive functioning and brain health at various points in the lifespan (Gunstad et al., 2007, 2008; Raji et al., 2010; Kennedy et al., 2016). As expected, our results confirm a previous study that included data from the YAHCP that showed that high BMI was associated with widespread reduced WM integrity (Repple et al., 2018). The novelty of the current findings is that by also including several structural markers of GM, we observed a fairly selective association between BMI and WM integrity that does not extend to GM, with the single exception in the right superior parietal lobe. Also, by including upper-body strength and submaximal cardiovascular endurance as additional predictors of interest, we show that the effects of BMI cannot be explained by these performancebased fitness measures, and further, that these fitness measures were not uniquely associated with brain structure. Finally, by allowing for non-linearities in these associations, we observed that in instances in which the association deviated from linear, the most common pattern was for the slope to be steepest over the range of BMI values representing normal through overweight (i.e., 18-30), after which the slope became shallower. This was most apparent for WM in the bilateral cerebral peduncle and bilateral hippocampal portion of the cingulum, and for the right superior parietal GM thickness.

WM integrity begins to decline in mid adulthood and it correlates with cognitive performance; furthermore, loss of WM integrity and other WM abnormalities are features of various dementia subtypes, including Alzheimer's disease (Wassenaar et al., 2019). Our findings are consistent with previous studies showing that although there is a negative association between 


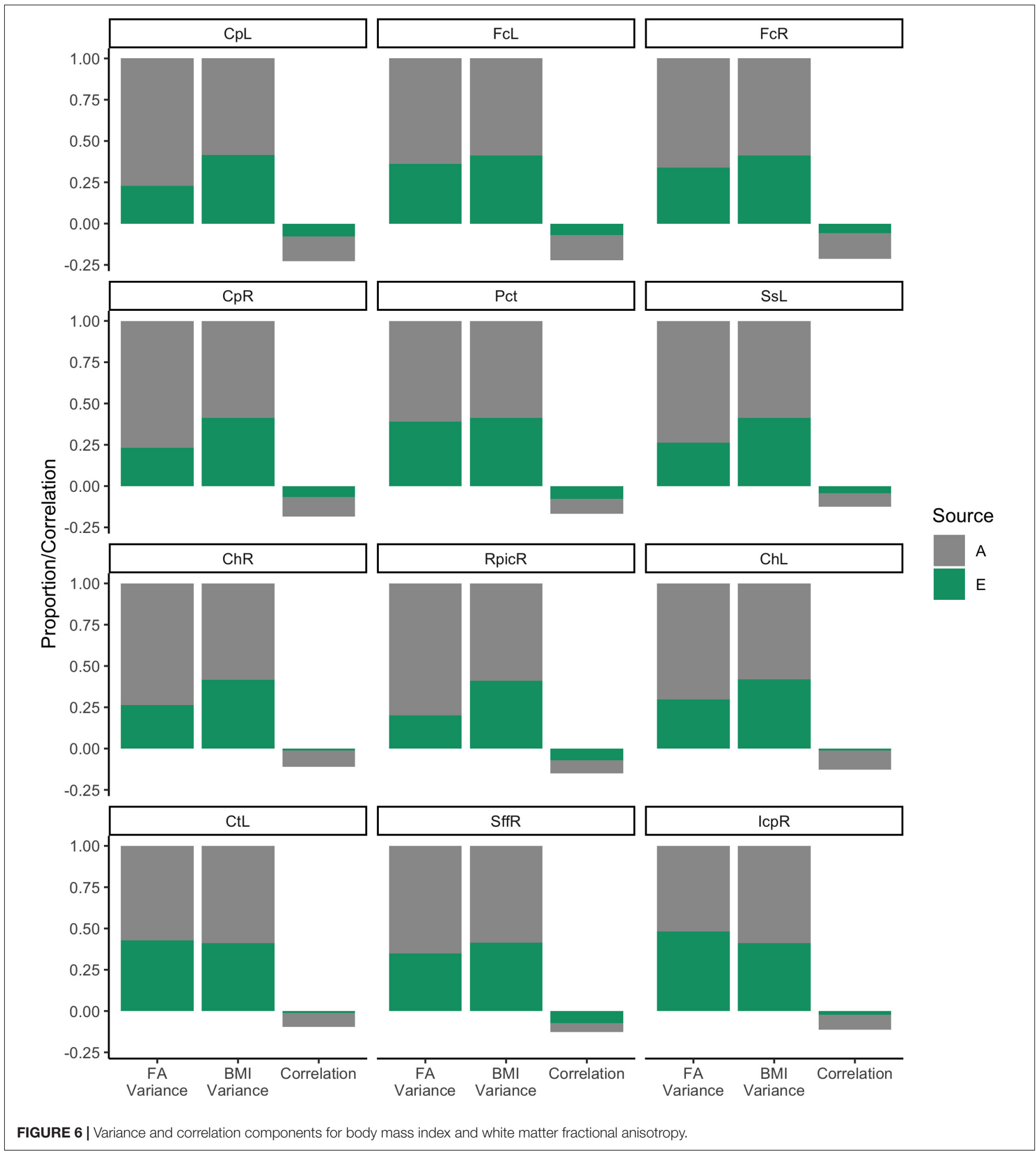

BMI and WM integrity throughout the brain, among the strongest statistical signals were subcortical brain stem pathways, including bilateral cerebral peduncle and pontine cross tract (Verstynen et al., 2012; Repple et al., 2018). By linking the midbrain to the thalamus, and to the cerebrum more generally, the cerebral peduncles are critically involved in motor and sensory functions, including motor control and coordination (Jane et al., 1968). Our findings also support previous studies showing a negative association between BMI and WM integrity in the fornix (Stanek et al., 2011; Xu et al., 2013). The fornix carries projection fibers from the hippocampus and is implicated in higher-order learning and memory (Kantarci, 2014). Decreased 


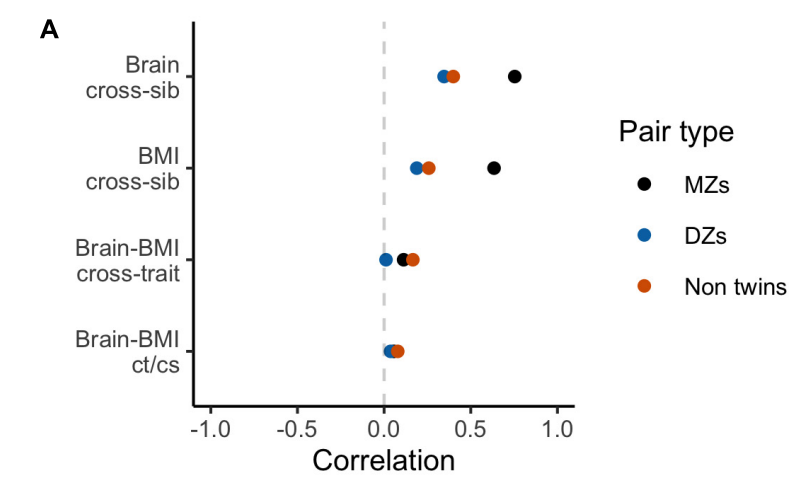

B

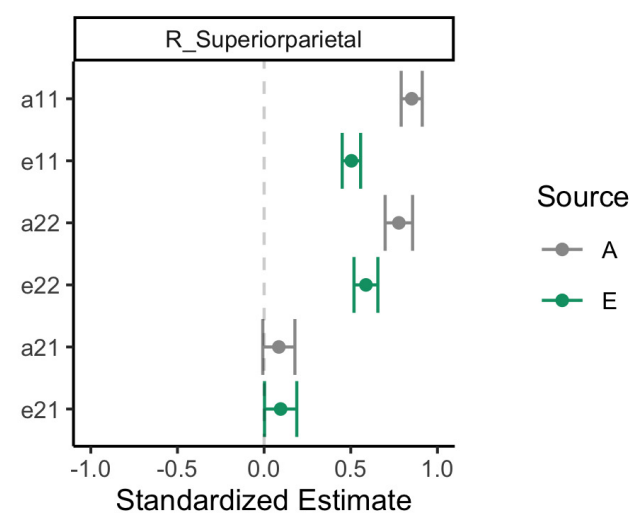

C

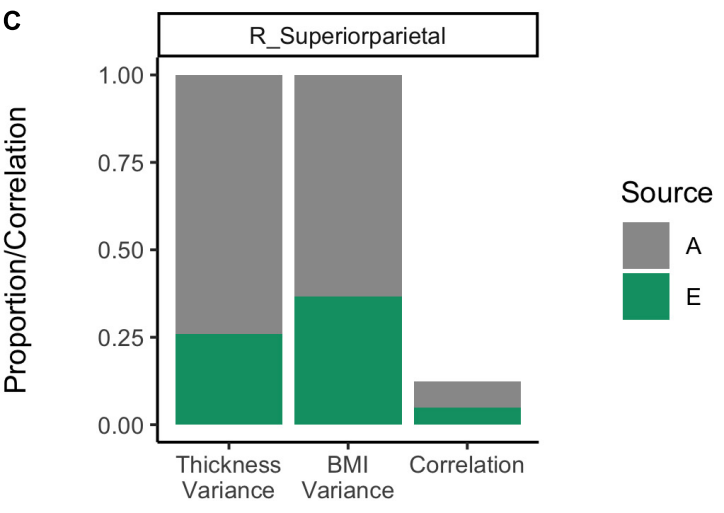

FIGURE 7 | Summary of behavioral genetic analyses of body mass index and right superior parietal thickness, including cross-sibling, cross trait correlations (A), path estimates from AE bivariate model (B), and variance and correlation components from AE bivariate model (C).

fornix FA is observed with increasing age, and in mild cognitive impairment and Alzheimer's disease (Kantarci, 2014). We also observed the BMI-FA association in the bilateral hippocampal portion of the cingulum, which has also been linked to memory functioning (Ezzati et al., 2016) and to Alzheimer's disease (Dalboni Da Rocha et al., 2020). In light of the association of BMI in midlife with risk of dementia in late life (Kivimaki et al., 2018), it is possible that these WM tracts may play some role in transmitting this effect.
Similar to a previous analysis of YA-HCP data (Vainik et al., 2018), we observed a positive association between BMI and right superior parietal thickness; however, unlike this previous analysis, we failed to observe associations within other GM regions. Reasons for this discrepancy may include a stricter significance threshold in the current study that did not detect the weaker effects reported previously and the larger set of covariates used in the current study, which notably included performance-based physical fitness, educational attainment, and annual income.

We also failed to observe associations between either submaximal cardiovascular endurance or grip strength with any of the markers of brain structure, which is inconsistent with a previous analysis of the YA-HCP data that found an association between endurance and WM integrity (Opel et al., 2019). However, the current analysis differed in its analytic approach by including a larger set of covariates and using an ROI-based, as opposed to a voxel-based, approach to analyzing the associations between BMI and neuroimaging data. The lack of associations between fitness and brain structure is also inconsistent with other studies that have explored these associations in youth (Chaddock-Heyman et al., 2015) and older adults (Oberlin et al., 2015). The inconsistency with the current results could arise for numerous reasons. It is possible that these associations are weaker during young adulthood as compared to during childhood development or aging. Previous studies (ChaddockHeyman et al., 2015; Oberlin et al., 2015) also used superior measures of fitness (e.g., direct assessment of maximal oxygen uptake during a graded exercise test), which may have provided better measurement of fitness, and in turn, of the true association with brain structure. It is also possible that the large sample size of the current study allowed us to avoid detecting false positive findings. An underappreciated phenomena is that small studies, in which constructs are measured with error (as is ubiquitous in the behavioral and neurocognitive sciences), are prone to overestimate effect sizes, leading to false positives, in addition to being prone to failure in detecting effects (Loken and Gelman, 2017). This is especially true when the publication of research findings is contingent of the findings being statistically significant. To resolve the inconsistencies of the current study with previous ones, large-sample, lifespan studies with goldstandard measures of physical fitness are needed; the result will be better estimation of the associations between fitness and brain structure and determination of how these associations might change over the life course.

\section{Behavioral Genetics of Body Composition and Brain Structure}

To further explore the nature of the association between BMI and brain structure, our study made use of the large set of twins and full siblings contained in the YA-HCP. Using behavioral genetic analyses, we estimated the degree to which variation in BMI and brain structure, and the covariation between BMI and brain structure, could be explained by heritability-i.e., a set of common genes that directly or indirectly impact BMI and brain structure-versus environmental effects. The first insight 
from these analyses was that a simpler behavioral genetic model, in which variation and covariation was partitioned into genetic versus environmental effects, fit the data no worse, despite being simpler, than a model which further apportioned environmental effects into twin-specific effects, more generally sibling shared effects, and non-shared environmental effects. Others have also found that this simpler model fits (co)variation in BMI and neurocognition well (Vainik et al., 2018; Wood et al., 2019). This would imply that shared environmental effects-whether specific to twins or to full siblings more generally-have little consistent contribution to any connections between BMI and brain structure.

The second insight from these behavioral genetic analyses is that there was a clear heritability effect underpinning variation in BMI and the identified brain structures, as well as the covariation between BMI and these brain structures. Indeed, this heritability effect exceeded the environmental effect across all variance and correlation estimates (see Figures 6, 7C) and comports with previous studies that have observed a clear genetic effect on variation in BMI and neurocognition analyzed independently (Friedman et al., 2008; Elks et al., 2012; Kochunov et al., 2015), as well as in studies that employed bivariate behavioral genetic analyses similar to the current study (Vainik et al., 2018; Wood et al., 2019).

This heritability effect is the portion of the variance or covariance that can be attributed to an additive genetic effect after adjusting for the covariates. Although heritability estimates provide some insight as to how various phenotypes (say, BMI and brain structure) are related, there is still a large degree of ambiguity about the nature of these associations. This clear heritability effect could reflect a common set of genes that causally affect both BMI and brain structure, socalled genetic confounding or pleiotropy (Vainik et al., 2018). If genetic confounding largely accounts for the association between BMI and brain structure, intervening upon either phenotype would be expected to have little impact on the other phenotype. Alternatively, genetic effects could directly affect one phenotype that in turn, through active selection of environments and behaviors, impacts the second phenotype (Scarr and Mccartney, 1983). If our heritability estimate truly reflects a cascading effect where genetic effects are transmitted through an intermediary phenotype, there is evidence in the literature that the cascade could flow in either direction. One possibility is that differences in brain structure impact obesogenic behaviors through decision-making processes, and in turn, leads to variation in BMI (AlonsoAlonso and Pascual-Leone, 2007; Lowe et al., 2019). Under this proposed directionality, it may be helpful to remediate decision-making processes in order to promote healthful behavior or structure the environment to support healthful behaviors and reduce the reliance of individual decisionmaking (Hall and Fong, 2015; Hall, 2016), all with the intent of reducing obesity. Alternatively, BMI might impact brain structure via cardiometabolic effects, including inflammation and hypertension (Williamson et al., 2018; Repple et al., 2019). Interventions to reduce BMI might have the added benefit of improving brain structure, especially WM integrity, though evidence from clinical trials is lacking (Wassenaar et al., 2019). It may also be worthwhile to intervene on the cardiometabolic consequences of excess weight. A recent clinical trial showed that intensive blood pressure treatment reduced the accrual of WM lesions over time among hypertensive adults 50 years or older (Nasrallah et al., 2019).

A final note from the behavioral genetic analyses is that although generally smaller in size, the environmental contributions to (co)variation in BMI and brain structure were not negligible. Thus, it is possible that environmental exposures (e.g., diet, physical activity) that operate outside of genetic selection of environments make a meaningful contribution to the association between body composition and brain structure.

\section{Limitations}

The current study has noteworthy limitations and the findings are conditioned on certain untested assumptions. The crosssectional study design prohibits us from understanding the causality or even directionality of the association between BMI and brain structure. As noted above, even a strong heritability effect is consistent with various causal models of the studied variables. Our physical fitness and body composition measures also have limitations. Physical fitness was measured less precisely than gold-standard measures, such as maximal oxygen uptake during a graded exercise test. BMI was calculated using selfreported height and weight, and further BMI is an imperfect proxy for actual body composition (e.g., percentage of body fat or amount of central adiposity), which have stronger negative cardiometabolic sequelae (Frankenfield et al., 2001). Our behavioral genetic analyses require the untested assumptions that there is no assortative mating (i.e., individuals are not mating with others with similar BMI or brain structure more than expected at random) and that the environmental effects are equivalent across sibling pair type.

\section{CONCLUSION}

Our study adds to a fairly consistent pattern of findings showing that higher BMI correlates with lower WM integrity at various points in the lifespan [summarized in Wassenaar et al. (2019)]. We also extend the current literature in other ways. By taking a comprehensive approach to analyzing fitness and body composition with several structural markers of brain health, we show that BMI, but not strength or endurance, uniquely correlates with WM integrity, and that there was very limited evidence for an association with GM structure. A final contribution of the current work stems from findings from our behavioral genetic analyses of twins and full siblings. These analyses confirm previous studies by showing that heritability contributes to a majority proportion of variation in BMI and brain structure. Furthermore, we show that generally at least $\frac{1}{2}$ of the correlation between BMI and brain structure can be attributed to heritability. This does not imply that either BMI or brain structure is immutable, but it does improve our understanding of the etiology of the association between BMI and brain structure. 


\section{DATA AVAILABILITY STATEMENT}

Publicly available datasets were analyzed in this study. This data can be found here: https://db.humanconnectome.org.

\section{ETHICS STATEMENT}

The studies involving human participants were reviewed and approved by Institutional Review Board at Simon Fraser University. The patients/participants provided their written informed consent to participate in this study.

\section{AUTHOR CONTRIBUTIONS}

JB conceptualized the study, acquired the data, conducted the statistical analyses, and wrote the first draft of the manuscript. ED contributed to analysis and interpretation of neuroimaging results. RC acquired and synthesized literature necessary to the Introduction and Discussion sections. TC

\section{REFERENCES}

Aberg, M. A., Pedersen, N. L., Toren, K., Svartengren, M., Backstrand, B., Johnsson, T., et al. (2009). Cardiovascular fitness is associated with cognition in young adulthood. Proc. Natl. Acad. Sci. U.S.A. 106, 20906-20911. doi: 10.1073/pnas. 0905307106

Alonso-Alonso, M., and Pascual-Leone, A. (2007). The right brain hypothesis for obesity. JAMA 297, 1819-1822. doi: 10.1001/jama.297.16.1819

Barch, D. M., Burgess, G. C., Harms, M. P., Petersen, S. E., Schlaggar, B. L., Corbetta, M., et al. (2013). Function in the human connectome: task-fMRI and individual differences in behavior. Neuroimage 80, 169-189. doi: 10.1016/j. neuroimage.2013.05.033

Best, J. R. (2020). Physical fitness and age-related differences in cognition and cortical thickness in young adulthood. Dev. Psychol. 56, 1984-1998. doi: 10. 1037/dev0001097

Bohannon, R. W., Magasi, S. R., Bubela, D. J., Wang, Y. C., and Gershon, R. C. (2012). Grip and knee extension muscle strength reflect a common construct among adults. Muscle Nerve 46, 555-558. doi: 10.1002/mus.23350

Cadenas-Sanchez, C., Esteban-Cornejo, I., Migueles, J. H., Labayen, I., VerdejoRoman, J., Mora-Gonzalez, J., et al. (2020). Differences in brain volume between metabolically healthy and unhealthy overweight and obese children: the role of fitness. J. Clin. Med. 9:1059. doi: 10.3390/jcm9041059

Chaddock-Heyman, L., Erickson, K. I., Kienzler, C., King, M., Pontifex, M. B., Raine, L. B., et al. (2015). The role of aerobic fitness in cortical thickness and mathematics achievement in preadolescent children. PLoS One 10:e134115. doi: 10.1371/journal.pone.0134115

Cole, R. C., Hazeltine, E., Weng, T. B., Wharff, C., Dubose, L. E., Schmid, P., et al. (2020). Cardiorespiratory fitness and hippocampal volume predict faster episodic associative learning in older adults. Hippocampus 30, 143-155. doi: 10.1002/hipo. 23151

Dalboni Da Rocha, J. L., Bramati, I., Coutinho, G., Tovar Moll, F., and Sitaram, R. (2020). Fractional anisotropy changes in parahippocampal cingulum due to Alzheimer's disease. Sci. Rep. 10:2660.

Ding, K., Tarumi, T., Zhu, D. C., Tseng, B. Y., Thomas, B. P., Turner, M., et al. (2018). Cardiorespiratory fitness and white matter neuronal fiber integrity in mild cognitive impairment. J. Alzheimers Dis. 61, 729-739. doi: 10.3233/jad170415

Elks, C. E., Den Hoed, M., Zhao, J. H., Sharp, S. J., Wareham, N. J., Loos, R. J., et al. (2012). Variability in the heritability of body mass index: a systematic review and meta-regression. Front. Endocrinol. 3:29. doi: 10.3389/fendo.2012.00029

Erickson, K., Raji, C., Lopez, O., Becker, J., Rosano, C., Newman, A., et al. (2010). Physical activity predicts gray matter volume in late adulthood: the contributed to the conceptualization and interpretation of the study. All authors provided critical feedback and editing to the submitted manuscript.

\section{FUNDING}

Data were provided by the Human Connectome Project, WUMinn Consortium (Principal Investigators: David Van Essen and Kamil Ugurbil; 1U54MH091657) funded by the $16 \mathrm{NIH}$ Institutes and Centers that support the NIH Blueprint for Neuroscience Research; and by the McDonnell Center for Systems Neuroscience at Washington University.

\section{SUPPLEMENTARY MATERIAL}

The Supplementary Material for this article can be found online at: https://www.frontiersin.org/articles/10.3389/fpsyg. 2020.608049/full\#supplementary-material

Cardiovascular Health Study. Neurology 75, 1415-1422. doi: 10.1212/wnl. 0b013e3181f88359

Erickson, K. I., Leckie, R. L., and Weinstein, A. M. (2014). Physical activity, fitness, and gray matter volume. Neurobiol. Aging 35(Suppl. 2), S20-S28.

Erickson, K. I., Prakash, R. S., Voss, M. W., Chaddock, L., Hu, L., Morris, K. S., et al. (2009). Aerobic fitness is associated with hippocampal volume in elderly humans. Hippocampus 19, 1030-1039. doi: 10.1002/hipo. 20547

Esteban-Cornejo, I., Mora-Gonzalez, J., Cadenas-Sanchez, C., ContrerasRodriguez, O., Verdejo-Roman, J., Henriksson, P., et al. (2019). Fitness, cortical thickness and surface area in overweight/obese children: the mediating role of body composition and relationship with intelligence. Neuroimage 186, 771-781. doi: 10.1016/j.neuroimage.2018.11.047

Ezzati, A., Katz, M. J., Lipton, M. L., Zimmerman, M. E., and Lipton, R. B. (2016). Hippocampal volume and cingulum bundle fractional anisotropy are independently associated with verbal memory in older adults. Brain Imaging Behav. 10, 652-659. doi: 10.1007/s11682-015-9452-y

Frankenfield, D. C., Rowe, W. A., Cooney, R. N., Smith, J. S., and Becker, D. (2001). Limits of body mass index to detect obesity and predict body composition. Nutrition 17, 26-30. doi: 10.1016/s0899-9007(00)00471-8

Friedman, N. P., Miyake, A., Young, S. E., Defries, J. C., Corley, R. P., and Hewitt, J. K. (2008). Individual differences in executive functions are almost entirely genetic in origin. J. Exp. Psychol. Gen. 137, 201-225. doi: 10.1037/0096-3445. 137.2.201

Gershon, R. C., Wagster, M. V., Hendrie, H. C., Fox, N. A., Cook, K. F., and Nowinski, C. J. (2013). NIH toolbox for assessment of neurological and behavioral function. Neurology 80(11 Suppl. 3), S2-S6.

Glasser, M. F., Sotiropoulos, S. N., Wilson, J. A., Coalson, T. S., Fischl, B., Andersson, J. L., et al. (2013). The minimal preprocessing pipelines for the Human Connectome Project. Neuroimage 80, 105-124. doi: 10.1016/j. neuroimage.2013.04.127

Gordon, B. A., Rykhlevskaia, E. I., Brumback, C. R., Lee, Y., Elavsky, S., Konopack, J. F., et al. (2008). Neuroanatomical correlates of aging, cardiopulmonary fitness level, and education. Psychophysiology 45, 825-838.

Grasby, K. L., Verweij, K. J. H., Mosing, M. A., Zietsch, B. P., and Medland, S. E. (2017). "Estimating heritability from twin studies," in Statistical Human Genetics: Methods and Protocols, 2nd Edn, ed. R. C. Elston (New York, NY: Humana Press), 171-194.

Gunstad, J., Paul, R. H, Cohen, R. A, Tate, D. F, Spitznagel, M. B, and Gordon, E. (2007). Elevated body mass index is associated with executive dysfunction in otherwise healthy adults. Compr. Psychiatry 48, 57-61. doi: 10.1016/j. comppsych.2006.05.001 
Gunstad, J., Paul, R. H., Cohen, R. A., Tate, D. F., Spitznagel, M. B., Grieve, S., et al. (2008). Relationship between body mass index and brain volume in healthy adults. Int. J. Neurosci. 118, 1582-1593. doi: 10.1080/00207450701392282

Hall, P., and Fong, G. T. (2015). Temporal self-regulation theory: a neurobiologically informed model for physical activity behavior. Front. Hum. Neurosci. 9:117. doi: 10.3389/fnhum.2015.00117

Hall, P. A. (2016). Executive-control processes in high-calorie food consumption. Curr. Dir. Psychol. Sci. 25, 91-98. doi: 10.1177/0963721415625049

Harrell, F. E. (2019a). Regression Modeling Strategies: With Applications to Linear Models, Logistic and Ordinal Regression, and Survival Analysis. Cham: Springer.

Harrell, F. E. (2019b). rms: Regression Modeling Strategies. R package Version 5.1-4. Available online at: https://CRAN.R-project.org/package=rms (accessed October 21, 2020).

Ho, A. J., Raji, C. A., Becker, J. T., Lopez, O. L., Kuller, L. H., Hua, X., et al. (2011). The effects of physical activity, education, and body mass index on the aging brain. Hum. Brain Mapp. 32, 1371-1382. doi: 10.1002/hbm.21113

Jane, J. A., Yashon, D., Becker, D. P., Beatty, R., and Sugar, O. (1968). The effect of destruction of the corticospinal tract in the human cerebral peduncle upon motor function and involuntary movements. J. Neurosurg. 29, 581-585. doi: 10.3171/jns.1968.29.6.0581

Kantarci, K. (2014). Fractional anisotropy of the fornix and hippocampal atrophy in Alzheimer's disease. Front. Aging Neurosci. 6:316. doi: 10.3389/fnagi.2014. 00316

Kennedy, J. T., Collins, P. F., and Luciana, M. (2016). Higher adolescent body mass index is associated with lower regional gray and white matter volumes and lower levels of positive emotionality. Front. Neurosci. 10:413. doi: 10.3389/fnins.2016. 00413

Kivimaki, M., Luukkonen, R., Batty, G. D., Ferrie, J. E., Pentti, J., Nyberg, S. T., et al. (2018). Body mass index and risk of dementia: analysis of individuallevel data from 1.3 million individuals. Alzheimers Dement. 14, 601-609. doi: 10.1016/j.jalz.2017.09.016

Kochunov, P., Jahanshad, N., Marcus, D., Winkler, A., Sprooten, E., Nichols, T. E., et al. (2015). Heritability of fractional anisotropy in human white matter: a comparison of Human Connectome Project and ENIGMA-DTI data. Neuroimage 111, 300-311. doi: 10.1016/j.neuroimage.2015.02.050

Loken, E., and Gelman, A. (2017). Measurement error and the replication crisis. Science 355, 584-585. doi: 10.1126/science.aal3618

Lowe, C. J., Reichelt, A. C., and Hall, P. A. (2019). The prefrontal cortex and obesity: a health neuroscience perspective. Trends Cogn. Sci. 23, 349-361. doi: 10.1016/j.tics.2019.01.005

Marks, B. L., Katz, L. M., Styner, M., and Smith, J. K. (2011). Aerobic fitness and obesity: relationship to cerebral white matter integrity in the brain of active and sedentary older adults. Br. J. Sports Med. 45, 1208-1215. doi: 10.1136/bjsm. 2009.068114

Moffitt, T. E., Belsky, D. W., Danese, A., Poulton, R., and Caspi, A. (2016). The longitudinal study of aging in human young adults: knowledge gaps and research agenda. J. Gerontol. A Biol. Sci. Med. Sci. 72, 210-215. doi: 10.1093/ gerona/glw191

Mori, S., Oishi, K., Jiang, H., Jiang, L., Li, X., Akhter, K., et al. (2008). Stereotaxic white matter atlas based on diffusion tensor imaging in an ICBM template. Neuroimage 40, 570-582. doi: 10.1016/j.neuroimage.2007.12.035

Nasrallah, I. M., Pajewski, N. M., Auchus, A. P., Chelune, G., Cheung, A. K., Cleveland, M. L., et al. (2019). Association of intensive vs standard blood pressure control with cerebral white matter lesions. JAMA 322, 524-534.

Nyholt, D. R. (2004). A simple correction for multiple testing for single-nucleotide polymorphisms in linkage disequilibrium with each other. Am. J. Hum. Genet. 74, 765-769. doi: 10.1086/383251

Oberlin, L. E., Verstynen, T. D., Burzynska, A. Z., Voss, M. W., Prakash, R. S., Chaddock-Heyman, L., et al. (2015). White matter microstructure mediates the relationship between cardiorespiratory fitness and spatial working memory in older adults. Neuroimage 131, 91-101. doi: 10.1016/j.neuroimage.2015.09.053

Oishi, K., Zilles, K., Amunts, K., Faria, A., Jiang, H., Li, X., et al. (2008). Human brain white matter atlas: identification and assignment of common anatomical structures in superficial white matter. Neuroimage 43, 447-457. doi: 10.1016/j. neuroimage.2008.07.009

Opel, N., Martin, S., Meinert, S., Redlich, R., Enneking, V., Richter, M., et al. (2019). White matter microstructure mediates the association between physical fitness and cognition in healthy, young adults. Sci. Rep. 9:12885.
Pierpaoli, C., Jezzard, P., Basser, P. J., Barnett, A., and Di Chiro, G. (1996). Diffusion tensor MR imaging of the human brain. Radiology 201, 637-648.

Raji, C. A., Ho, A. J., Parikshak, N. N., Becker, J. T., Lopez, O. L., Kuller, L. H., et al. (2010). Brain structure and obesity. Hum. Brain Mapp. 31, 353-364.

Repple, J., Karliczek, G., Meinert, S., Forster, K., Grotegerd, D., Goltermann, J., et al. (2019). Variation of HbA1c affects cognition and white matter microstructure in healthy, young adults. Mol. Psychiatry doi: 10.1038/s41380-019-0504-3

Repple, J., Opel, N., Meinert, S., Redlich, R., Hahn, T., Winter, N. R., et al. (2018). Elevated body-mass index is associated with reduced white matter integrity in two large independent cohorts. Psychoneuroendocrinology 91, 179-185. doi: 10.1016/j.psyneuen.2018.03.007

Reuben, D. B., Magasi, S., Mccreath, H. E., Bohannon, R. W., Wang, Y.-C., Bubela, D. J., et al. (2013). Motor assessment using the NIH toolbox. Neurology 80(11 Suppl. 3), S65-S75.

Rodriguez-Ayllon, M., Esteban-Cornejo, I., Verdejo-Roman, J., Muetzel, R. L., Mora-Gonzalez, J., Cadenas-Sanchez, C., et al. (2020). Physical fitness and white matter microstructure in children with overweight or obesity: the ActiveBrains project. Sci. Rep. 10:12469.

Scarr, S., and Mccartney, K. (1983). How people make their own environments: a theory of genotype $\rightarrow$ environment effects. Child Dev. 54, 424-435. doi: $10.2307 / 1129703$

Sexton, C. E., Betts, J. F., Demnitz, N., Dawes, H., Ebmeier, K. P., and JohansenBerg, H. (2016). A systematic review of MRI studies examining the relationship between physical fitness and activity and the white matter of the ageing brain. Neuroimage 131, 81-90. doi: 10.1016/j.neuroimage.2015.09.071

Smith, S. M., Jenkinson, M., Johansen-Berg, H., Rueckert, D., Nichols, T. E., Mackay, C. E., et al. (2006). Tract-based spatial statistics: voxelwise analysis of multi-subject diffusion data. Neuroimage 31, 1487-1505. doi: 10.1016/j. neuroimage.2006.02.024

Stanek, K. M., Grieve, S. M., Brickman, A. M., Korgaonkar, M. S., Paul, R. H., Cohen, R. A., et al. (2011). Obesity is associated with reduced white matter integrity in otherwise healthy adults. Obesity 19, 500-504. doi: 10.1038/oby. 2010.312

Tseng, B. Y., Gundapuneedi, T., Khan, M. A., Diaz-Arrastia, R., Levine, B. D., Lu, H., et al. (2013). White matter integrity in physically fit older adults. Neuroimage 82, 510-516. doi: 10.1016/j.neuroimage.2013.06.011

Tucker-Drob, E. M. (2019). Cognitive aging and dementia: a life-span perspective. Annu. Rev. Dev. Psychol. 1, 177-196. doi: 10.1146/annurev-devpsych-121318085204

Vainik, U., Baker, T. E., Dadar, M., Zeighami, Y., Michaud, A., Zhang, Y., et al. (2018). Neurobehavioral correlates of obesity are largely heritable. Proc. Natl. Acad. Sci. U.S.A. 115, 9312-9317. doi: 10.1073/pnas.1718206115

Van Essen, D. C., Smith, S. M., Barch, D. M., Behrens, T. E., Yacoub, E., Ugurbil, K., et al. (2013). The WU-Minn Human Connectome project: an overview. Neuroimage 80, 62-79. doi: 10.1016/j.neuroimage.2013.05.041

Van Essen, D. C., Ugurbil, K., Auerbach, E., Barch, D., Behrens, T. E., Bucholz, R., et al. (2012). The Human Connectome project: a data acquisition perspective. Neuroimage 62, 2222-2231.

Verstynen, T. D., Weinstein, A. M., Schneider, W. W., Jakicic, J. M., Rofey, D. L., and Erickson, K. I. (2012). Increased body mass index is associated with a global and distributed decrease in white matter microstructural integrity. Psychosom. Med. 74, 682-690. doi: 10.1097/psy.0b013e318261909c

Wassenaar, T. M., Yaffe, K., Van Der Werf, Y. D., and Sexton, C. E. (2019). Associations between modifiable risk factors and white matter of the aging brain: insights from diffusion tensor imaging studies. Neurobiol. Aging 80, 56-70. doi: 10.1016/j.neurobiolaging.2019.04.006

Weintraub, S., Dikmen, S., Heaton, R. K., Tulsky, D., Zelazo, P. D., Bauer, P. J., et al. (2013). Cognition assessment using the NIH toolbox. Neurology 80(11 Suppl. 3), S54-S64.

Williams, V. J., Hayes, J. P., Forman, D. E., Salat, D. H., Sperling, R. A., Verfaellie, M., et al. (2017). Cardiorespiratory fitness is differentially associated with cortical thickness in young and older adults. Neuroimage 146, 1084-1092. doi: 10.1016/j.neuroimage.2016.10.033

Williamson, W., Lewandowski, A. J., Forkert, N. D., Griffanti, L., Okell, T. W., Betts, J., et al. (2018). Association of cardiovascular risk factors with MRI indices of cerebrovascular structure and function and white matter hyperintensities in young adults. JAMA 320, 665-673. doi: 10.1001/jama.2018. 11498 
Winkler, A. M., Kochunov, P., Blangero, J., Almasy, L., Zilles, K., Fox, P. T., et al. (2010). Cortical thickness or grey matter volume? The importance of selecting the phenotype for imaging genetics studies. Neuroimage 53, 1135-1146. doi: 10.1016/j.neuroimage.2009.12.028

Wood, A. C., Vainik, U., Engelhardt, L. E., Briley, D. A., Grotzinger, A. D., Church, J. A., et al. (2019). Genetic overlap between executive functions and BMI in childhood. Am. J. Clin. Nutr. 110, 814-822. doi: 10.1093/ajcn/ nqz109

Wood, K. N., Nikolov, R., and Shoemaker, J. K. (2016). Impact of long-term endurance training vs. guideline-based physical activity on brain structure in healthy aging. Front. Aging Neurosci. 8:155. doi: 10.3389/fnagi.2016.00155

Xu, J., Li, Y., Lin, H., Sinha, R., and Potenza, M. N. (2013). Body mass index correlates negatively with white matter integrity in the fornix and corpus callosum: a diffusion tensor imaging study. Hum. Brain Mapp. 34, 1044-1052. doi: $10.1002 / \mathrm{hbm} .21491$

Conflict of Interest: The authors declare that the research was conducted in the absence of any commercial or financial relationships that could be construed as a potential conflict of interest.

Copyright (c) 2020 Best, Dao, Churchill and Cosco. This is an open-access article distributed under the terms of the Creative Commons Attribution License (CC BY). The use, distribution or reproduction in other forums is permitted, provided the original author(s) and the copyright owner(s) are credited and that the original publication in this journal is cited, in accordance with accepted academic practice. No use, distribution or reproduction is permitted which does not comply with these terms. 